\title{
Impact of a system-wide multicomponent intervention on administrative diagnostic coding for delirium and other cognitive frailty syndromes: observational prospective study
}

\author{
Authors: Sarah T Pendlebury, ${ }^{A}$ Nicola G Lovett, ${ }^{B}$ Ross ] Thomson ${ }^{C}$ and Sarah C Smith ${ }^{D}$
}

\begin{abstract}
Background
We determined the impact of a system-wide multicomponent intervention to improve recognition and documentation of cognitive frailty syndromes on hospital administrative coding for delirium.
\end{abstract}

Methods

A multicomponent intervention including introduction of structured patient assessment including cognitive/delirium screen, regular audit/feedback and educational seminars was undertaken (2012-17). Sensitivity and specificity of administrative International Classification of Diseases, 10th revision (ICD-10) delirium codes for the gold standard of prospectively clinically diagnosed delirium were calculated in consecutive patients admitted to acute medicine over five 8-week cycles (2010-18).

Results

Among 1,281 consecutive unselected admissions to acute medicine overall (mean / standard deviation age $=70.0 / 19.2$ years; $n=615$ ( $48.0 \%$ ) male), 320 had clinical delirium diagnosis ( $n=220$ delirium only; $n=100$ delirium on dementia). Sensitivity of delirium coding increased from $12.8 \%$ (95\% confidence interval (CI) 5.6-26.7) in 2010 to $60.2 \%$ (95\% CI $50.1-69.7$; $\left.p_{\text {trend }}<0.0001\right)$ in 2018 while specificity remained at $>99 \%$ throughout.

Conclusion

A multicomponent intervention increased sensitivity of hospital administrative diagnostic coding for delirium almost six-fold without increasing the false positive diagnosis rate.

Authors: ${ }^{\text {A }}$ consultant in acute general (internal) medicine and geriatrics and associate professor, Centre for Prevention of Stroke and Dementia, Oxford, UK and NIHR Oxford Biomedical Research Centre, Oxford, UK; ${ }^{B}$ consultant in acute general (internal) medicine and geriatrics, Oxford University Hospitals NHS Foundation Trust,

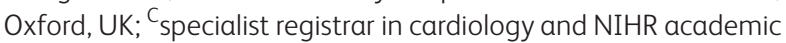
clinical fellow, Royal Free London NHS Foundation Trust, London, UK and Queen Mary University of London, London, UK; ${ }^{\mathrm{D}}$ consultant in acute general (internal) medicine and geriatrics, Oxford University Hospitals NHS Foundation Trust, Oxford, UK
KEYWORDS: Delirium, dementia, cognitive frailty, ICD-10 diagnostic coding, sensitivity/specificity

DOI: $10.7861 /$ clinmed.2019-0470

\section{Introduction}

Delirium affects one-fifth of acute general medicine patients with rates of over $40 \%$ in the oldest age bracket. ${ }^{1,2}$ Delirium is associated with high nursing needs, increased costs, death, dependency and future dementia. ${ }^{1,2}$ Recognition and diagnosis of delirium is important to facilitate individualised patient care, including communication with patients and families, mitigation of symptoms and avoidance of complications, discharge planning, and flagging of future dementia/delirium risks to primary care. ${ }^{1,2}$ However, clinical staff may have difficulty in recognising delirium owing to its transient and fluctuating nature and an emphasis on the physical aspects of disease. ' Unsurprisingly, therefore, the few available published data suggest that hospital administrative diagnostic coding for delirium substantially underestimate the true delirium rate. ${ }^{3-7}$

International Classification of Diseases, 10th revision (ICD-10) diagnostic coded data are used in commissioning, remuneration, quality assurance, audit and benchmarking/adjusting for casemix and also form the basis of Hospital Episode Statistics (HES) which are increasingly used in large-scale epidemiologic research and 'big data' studies. ${ }^{8-10}$ Lack of coding for delirium and other cognitive frailty syndromes will therefore lead to reduced hospital reimbursement payments (tariff), suboptimal casemix adjustment, commissioning and service planning, as well as under-estimation of the cognitive frailty burden in hospital cohorts. $^{9}$

We therefore carried out a system-wide multicomponent intervention (2012 onwards) within the Oxford University Hospitals NHS Foundation Trust (OUHFT) to improve clinical identification and documentation, and administrative coding, of cognitive frailty including delirium as part of a programme of work to improve the process of care of patients with comorbid cognitive disorders. ${ }^{2,11-14}$ The intervention included structured acute patient assessment and documentation, regular educational seminars, 
implementation of a trust-wide dementia strategy, and liaison with the coding team.

In the current observational prospective study, we aimed to determine the impact of the multicomponent intervention on hospital administrative coding for delirium and, specifically, whether delirium coding sensitivity was improved without sacrificing specificity. ICD-10 diagnostic coded data for delirium obtained from the administrative coding team were compared with the gold standard clinical delirium diagnosis acquired prospectively in acute general medicine patients from 2010-18. We also examined the sensitivity and specificity of dementia ICD-10 coding and the use of non-specific ICD codes for confusion or altered behaviour over the same period for comparison.

\section{Methods}

\section{Patient cohorts}

The OUHFT provides secondary care services for a population of approximately 660,000 . The OUHFT acute general medicine department manages all adults with no separate admissions system for older patients. All consecutive unselected admissions to one acute medicine team over five 8-week cycles (SeptemberNovember 2010, April-June 2012, October-December 2015, June-July 2016 and November 2017-January 2018) were included in the current study. There were no exclusion criteria.

The methodology for prospective patient assessment and delirium diagnosis has been previously described elsewhere. ${ }^{2,11-14}$ However, briefly, all patients were seen within 24 hours of admission by an experienced consultant physician dually accredited in acute general (internal) medicine and geriatrics who was responsible for the patient's care, and at least every other day thereafter. On admission, patients aged $\geq 70$ years, or those with confusion, altered behaviour or 'brain at risk' (eg history of stroke, alcohol excess or Parkinson's disease) had a cognitive screen which included the Confusion Assessment Method (CAM) and a cognitive test (abbreviated mental test score (AMT) or mini-mental state examination (MMSE; 2010 only)) administered by the admitting junior doctor, trained in the use of the cognitive screen (see supplementary material S1: Figs S1 and S2). ${ }^{2,11-17}$ Delirium diagnosis was made according to Diagnostic and Statistical Manual of Mental Disorders, 4th edn (DSM-IV) criteria by the responsible physician after discussion with the medical team. $^{18}$

Demographic and clinical data, including pre-admission diagnosis of dementia, were recorded from the patient, relatives and medical records. Cognitive frailty sub-types were defined as delirium only, delirium superimposed on dementia, and dementia only as these are commonly used terms in clinical practice corresponding to existing ICD-10 coding categories. ${ }^{8,19}$ Delirium was categorised as prevalent delirium (on admission or occurring within the first 48 hours), incident delirium (occurring after the first 48 hours) or any delirium (occurring at any point during admission). 2,12,13

Relevant anonymised data were entered into the Oxford Cognitive Comorbidity, Frailty and Ageing Research Database (ORCHARD). ORCHARD was designed to facilitate observational studies, audit and service improvement in older and frail patients. Some data from the earlier cycles (2010-12) have already been published. ${ }^{2,12,13}$ ORCHARD is approved by the local regional ethics committee (COREC 18/SC/0184).

\section{Hospital administrative diagnostic ICD-10 coded data}

Hospital administrative diagnostic coding was undertaken by OUHFT coding staff after patient discharge according to standard practice without input from the clinical team. Diagnostic (ICD-10) coding data for the relevant admission were extracted from the electronic patient record (EPR) by the Oxford University Hospitals information team. ${ }^{8}$ ICD-10 codes were then searched for:

> any delirium code: F05.9 (delirium unspecified); F05.1 (delirium superimposed on dementia); F05.0 (delirium not superimposed on dementia); F05.8 (other delirium); and any other delirium code

> any dementia code: F03.X (dementia unspecified); F01.9 (vascular dementia); F05.1 (delirium superimposed on dementia); F00.9 (unspecified dementia in Alzheimer's disease); G30.9 (Alzheimer's disease); and any other dementia code

> any relevant ICD-10 symptom/sign code: including R41.0 (disorientation unspecified); R41.8 (other and unspecified symptoms involving cognitive function and awareness); R44.1 (visual hallucinations); R44.3 (hallucinations unspecified); and R45.1 (restlessness and agitation)

> F06.7 (mild cognitive disorder).

\section{System-wide multicomponent intervention}

The different elements of the multicomponent intervention to improve recognition and documentation of delirium and other cognitive frailty syndromes are shown in Fig 1. The intervention included seven components.

> Introduction of routine cognitive screening via a structured clerking pro forma. The cognitive screen (AMTS and CAM for delirium) and clerking pro forma was designed then piloted in 2010, rolled out in September 2013 (supplementary material S1: Fig S1) and introduced into the EPR (Cerner Millennium) in January 2015 (supplementary material S1: Fig S2). 2,15,16 The screen fires automatically in EPR to prompt completion at patient admission.

> Educational updates on delirium were delivered face-to-face to the coding team (2012, 2016 and 2017) including on the revised Charlson Comorbidity Index including 'delirium superimposed on dementia.' ${ }^{19-21}$

> Regular seminars were delivered to internal medicine and geratology medical, nursing and allied healthcare professionals (2013-present) with one-off seminars to other medical and surgical specialties. Inductions of new clinical staff include information on the cognitive screen and teaching on delirium/dementia is included in the junior doctor teaching programme.

> Although formal evaluation of educational sessions was not obtained for logistical reasons, feedback from the coding team regarding issues around the use of non-specific terms, such as 'confused', and lack of a structured patient assessment was used to inform the design of the clerking pro forma and the educational sessions delivered to clinicians.

> Rates of cognitive frailty including delirium in acute medicine 2010 onwards were highlighted at governance meetings, OUHFT board meetings and in the OUHFT dementia strategy.

> OUHFT dementia strategy (2014 and 2017) included the need for routine identification and documentation of cognitive frailty including delirium in older inpatients. 
Clerking pro forma with cognitive screen pilot

2011

2012

2013

$\begin{array}{ll}\text { Validation of } & \text { Cognitive screening } \\ \text { cognitive screen } & \text { education programme }\end{array}$

of rolling seminars for:

Clerking foundation doctors

pro forma core medical trainee

with cognitive doctors

screen roll-out registrars

Cognitive screen consultants

ntroduced into

the electronic allied health

patient record

2016

2017
Cyclical audit of rates of cognitive comorbidity in AGM

\section{Meeting with Cyclical audit of} coding team to rates of cognitive improve coding comorbidity in of delirium AGM

.

AGM

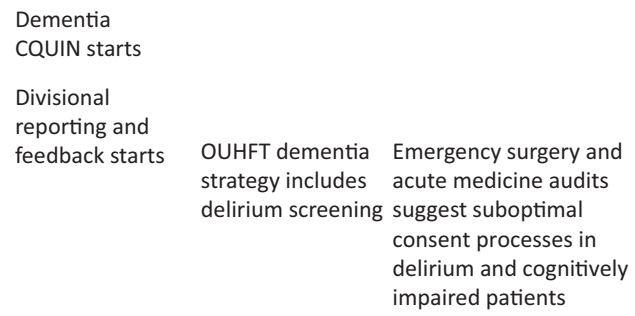

Dementia

CQUIN starts

Divisional

reporting and

feedback starts OUHFT dementia Emergency surgery and strategy includes acute medicine audits delirium screening suggest suboptimal consent processes in delirium and cognitively impaired patients

OUHFT dementia strategy updated

Dementia CQUIN stops

OUHFT consent form updated to include a prompt regarding cognitive screen and the need for capacity assessment

Fig 1. Different elements of the system-wide multicomponent intervention to improve identification and documentation of delirium and other comorbid cognitive disorders. AGM = acute general medicine; CQUIN = Commissioning for Quality and Innovation; OUHFT = Oxford University Hospitals NHS Foundation Trust.

$>$ Divisional reporting of cognitive screening rates to trust board (2013-present) was done as part of the dementia and delirium strategy and to fulfil national requirements (national dementia Commissioning for Quality and Innovation 2012-16; NHS England Dementia Assessment and Referral 2016-present). ${ }^{22}$

\section{Statistical analyses and reporting standards}

Sensitivity, specificity, positive predictive values (ppv) and negative predictive values (npv) for ICD-10 coding for delirium and for dementia vs the gold standard clinical diagnosis were calculated for each study cycle. Change in sensitivity of coding across study cycles from 2010-17/18 was assessed using the chi-squared test for trend. Clinical characteristics of patients with coded vs uncoded delirium and coded vs uncoded dementia were compared using analysis of variance (ANOVA) or chisquared test as appropriate for all study cycles combined. In patients with clinical diagnosis of delirium, independent associates of delirium coding were determined using logistic regression including age, sex, dementia diagnosis and total number of overall ICD-10 codes in the model for all study cycles combined and for each study cycle separately. Similar analyses were undertaken to determine the independent associates of dementia coding.

We evaluated the trust-wide impact of the multicomponent intervention, after completion of our study, by examining the proportion of total OUHFT adult acute medicine admissions coded for delirium between 01 July 2019 and 31 December 2019, and comparing these data with the proportion of all admissions coded for delirium in the $2017 / 18$ cohort in our study.
This paper was written in accordance with the Standards for Quality Improvement Reporting Excellence (SQUIRE 2.0) and the Strengthening the Reporting of Observational Studies in Epidemiology (STROBE) statement: guidelines for reporting observational studies. ${ }^{23,24}$

\section{Patient and public involvement}

ORCHARD was developed in collaboration with a patient and public involvement (PPI) group comprising prior inpatients, carers for people with cognitive frailty and a retired general practitioner. The reporting plan for the current study was discussed at the PPI meeting held in January 2019.

\section{Results}

Among 1,281 patients (mean / standard deviation (SD) age $=$ 70.0/19.2 years (range $16-102) ; n=615(48.0 \%)$ male) admitted over the five cycles (2010-2018), 320 patients had clinically diagnosed delirium ( $n=220$ delirium only; $n=100$ delirium on dementia), and 64 had dementia only (Table 1; Fig 2). Patients with clinically diagnosed delirium or dementia were older than the cohort overall (mean/SD age $=81.3 / 11.5$ and $84.5 / 8.5$ vs 70.0/19.2 years; Table 1) and had more diagnostic/symptom ICD-10 codes (mean/ SD codes $=8.6 / 3.4$ and 8.1/3.4 vs 6.5/6.5; Table 1 ).

The sensitivity of administrative ICD-10 coding for delirium for clinical delirium diagnosis increased over time from 12.8\% (95\% confidence interval (CI) 5.6-26.7\%) in 2010 to $60.2 \%$ (50.1-69.7\%) in 2018, $p_{\text {trend }}<0.0001$ (Table 2; Fig 3). The proportion of both prevalent and incident delirium cases coded for delirium also rose 
Table 1. Demographic details for the cohort and for patients with clinical diagnosis of delirium and dementia and ICD-10 coding status

\begin{tabular}{|c|c|c|c|c|c|c|c|c|c|}
\hline & $\begin{array}{l}\text { All } \\
n=1,281\end{array}$ & $\begin{array}{l}\text { All delirium } \\
n=320\end{array}$ & $\begin{array}{l}\text { Delirium } \\
\text { coded } \\
\mathrm{n}=111\end{array}$ & $\begin{array}{l}\text { Delirium } \\
\text { not coded } \\
n=209\end{array}$ & p value ${ }^{a}$ & $\begin{array}{l}\text { All dementia } \\
n=164\end{array}$ & $\begin{array}{l}\text { Dementia } \\
\text { coded } \\
n=103\end{array}$ & $\begin{array}{l}\text { Dementia } \\
\text { not coded } \\
n=61\end{array}$ & $\begin{array}{l}p \\
\text { value }^{b}\end{array}$ \\
\hline Age, mean/SD, years & 70.0/19.2 & $81.3 / 11.5$ & $83.1 / 8.2$ & $80.2 / 12.8$ & 0.04 & $84.5 / 8.5$ & $84.5 / 9.0$ & $84.4 / 7.7$ & 0.91 \\
\hline Age $<65$ years, $n(\%)$ & $411(32.1)$ & $26(8.1)$ & $2(1.8)$ & $24(11.5)$ & $0.01^{c}$ & $2(1.2)$ & $1(1.0)$ & $1(1.6)$ & $0.89^{c}$ \\
\hline Age $65-74$ years, $n(\%)$ & $205(16.0)$ & $37(11.6)$ & $15(13.8)$ & $22(10.5)$ & & $15(9.1)$ & $10(9.7)$ & $5(8.2)$ & \\
\hline Age $>74$ years, $n(\%)$ & 665 (51.9) & $257(80.3)$ & $94(84.7)$ & $163(78.0)$ & & $147(89.6)$ & $92(89.3)$ & $55(90.2)$ & \\
\hline Male, $n(\%)$ & $615(48.0)$ & $130(40.9)$ & $43(39.4)$ & $87(41.6)$ & 0.69 & $67(40.9)$ & $41(39.8)$ & $26(42.6)$ & 0.72 \\
\hline Dementia, n (\%) & $164(12.8)$ & $100(31.1)$ & $38(34.9)$ & $62(29.1)$ & 0.29 & $164(100)$ & $103(100)$ & $61(100)$ & $\mathrm{n} / \mathrm{a}$ \\
\hline $\begin{array}{l}\text { Number of ICD-10 } \\
\text { codes, mean/SD }\end{array}$ & $6.5 / 6.5$ & $8.6 / 3.4$ & $10.2 / 2.9$ & $7.8 / 3.4$ & $<0.0001$ & $8.1 / 3.4$ & $8.7 / 3.1$ & $7.0 / 3.6$ & 0.002 \\
\hline Any delirium, n (\%) & $320(24.9)$ & $320(100)$ & $111(100)$ & $209(100)$ & $\mathrm{n} / \mathrm{a}$ & $100(61.0)$ & $65(63.1)$ & $35(57.4)$ & 0.47 \\
\hline Prevalent delirium, n (\%) & $260(20.3)$ & $260(80.7)$ & $94(86.2)$ & 166 (77.9) & 0.07 & $86(52.4)$ & $57(55.3)$ & $29(47.5)$ & 0.33 \\
\hline Incident delirium, $\mathrm{n}(\%)$ & $120(9.4)$ & $120(37.3)$ & $35(32.1)$ & $85(39.9)$ & 0.17 & $34(20.7)$ & $24(23.3)$ & $10(16.4)$ & 0.29 \\
\hline $\begin{array}{l}\text { Both incident and } \\
\text { prevalent delirium, } \mathrm{n}(\%)\end{array}$ & $60(4.7)$ & $60(18.6)$ & $22(20.1)$ & $38(17.8)$ & 0.65 & $20(12.2)$ & $16(15.5)$ & $4(6.6)$ & 0.09 \\
\hline
\end{tabular}

${ }^{a}=$ delirium coded vs not coded; ${ }^{b}=$ dementia coded vs not coded; ${ }^{c}=$ chi-squared across age groups; ICD-10 = International Classification of Diseases, 10 th revision; $\mathrm{n} / \mathrm{a}=$ not applicable; $\mathrm{SD}=$ standard deviation.

over the five study cycles: $3 / 21,3 / 49,14 / 62,20 / 39,54 / 89$ and $5 / 32,4 / 15,8 / 32,7 / 19,11 / 22$, respectively. Only $2 / 959$ of patients without a clinical delirium diagnosis throughout the study period were allocated a delirium ICD-10 code, one of whom had dementia (Table 3). Specificity of administrative coding for delirium for clinical delirium diagnosis was therefore high throughout the study period

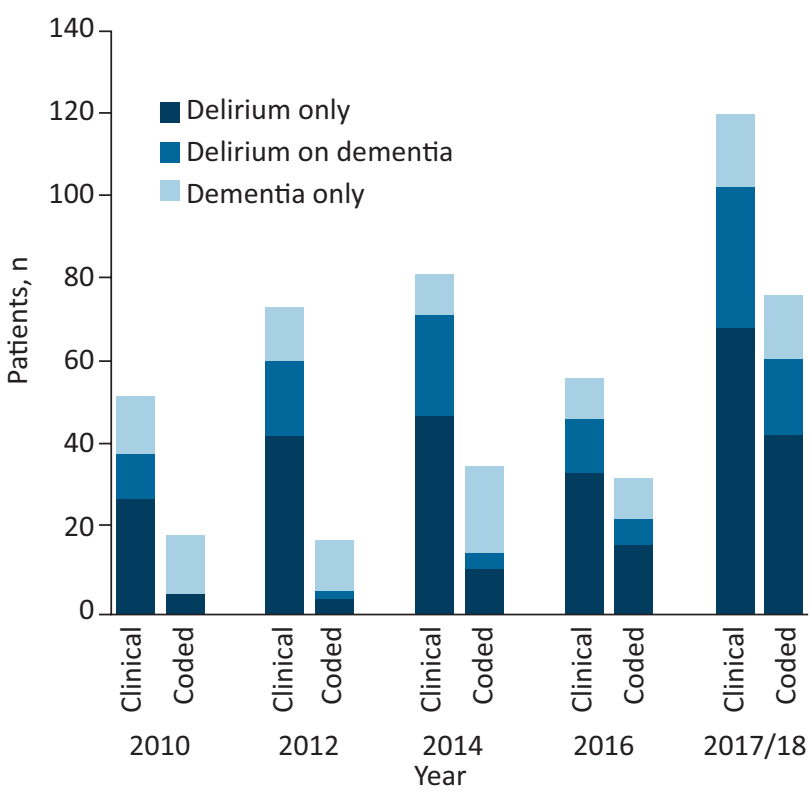

Fig 2. Clinical diagnosis and ICD-10 coded diagnosis of delirium only, delirium on dementia and dementia only for each study cycle. ICD-10 = International Classification of Diseases, 10 th revision. at over $99 \%$ (Table 2). Positive predictive value for delirium was also high ranging from $95.8 \%$ (76.2-99.4) to $100 \%$ (Table 2) but npv was lower, ranging from $77.7 \%(76.3-79.1)$ to $85.7 \%(84.1-87.1)$.

Sensitivity of administrative coding for dementia for clinically recorded dementia diagnosis rose non-significantly over the study period from $56 \%$ (34.9-75.6) in 2010 to $66.7 \%$ (52.1-79.2) in 2018 although rates were highest at $73.5 \%$ (55.6-87.1) in 2015, $\mathrm{p}_{\text {trend }}=0.13$. No patient had a dementia code in the absence of clinical recording of dementia diagnosis and specificity of dementia coding remained at $100 \%$ throughout the study period (Table 2). Positive predictive value for dementia coding was $100 \%$ throughout the study period with npv ranging from $92.5 \%$ (90.5-94.7) to $96.7 \%$ (94.3-98.1; Table 2).

Among the group of patients with clinically diagnosed delirium, those who received an ICD-10 delirium code were older than uncoded patients (mean $/ \mathrm{SD}$ age $=83.1 / 8.2$ years vs $80.2 / 12.8$ years; $p=0.04)$ and had more diagnostic codes overall (10.2/2.9 vs 7.8/3.4; $p<0.0001$; Table 1). However, when age, sex, dementia and number of diagnostic codes were entered in a regression model, only the number of diagnostic codes remained significant (odds ratio (OR) 1.25 (1.16-1.36); $p<0.0001$ ). Dementia coding in patients with clinically recorded dementia diagnosis was also associated with the overall number of diagnostic codes after adjustment for age and sex and delirium (OR 1.17 (1.06-1.29); $p=0.003)$. The association between number of diagnosis codes and delirium and dementia coding in those with the relevant clinically diagnosed syndrome was maintained throughout the study period (data not shown).

In the 111 patients with delirium who were coded, only 14 had a primary diagnosis ICD-10 code for delirium, the remainder being secondary diagnoses. The most commonly used code was F05.9 (delirium unspecified; $n=75$ ), followed by F05.1 (delirium superimposed on dementia; n=17), F05.0 (delirium not superimposed 
Table 2. Sensitivity, specificity, positive predictive values and negative predictive values of ICD-10 coding for delirium and dementia vs clinical diagnosis of delirium and recording of dementia diagnosis

\begin{tabular}{|c|c|c|c|c|c|c|}
\hline & \multicolumn{6}{|l|}{ Study cycle year } \\
\hline & 2010 & 2012 & 2015 & 2016 & 2018 & $p$ value \\
\hline \multicolumn{7}{|c|}{ Delirium coding } \\
\hline sensitivity & $12.8(4.3-27.4)$ & $9.8(3.7-20.2)$ & $20.8(12.2-32.0)$ & $48.9(34.1-63.9)$ & $60.2(50.1-69.7)$ & $<0.0001$ \\
\hline specificity & $100(98.2-100)$ & $100(99.1-100)$ & $100(98.4-100)$ & $99.2(95.4-100)$ & $99.6(97.5-100)$ & ns \\
\hline ppv & 100 & 100 & 100 & $95.8(76.2-99.4)$ & $98.4(89.7-99.8)$ & ns \\
\hline npv & $85.7(84.1-87.1)$ & $77.7(76.3-79.1)$ & 79.7 (77.7-81.6) & $83.0(78.7-86.6)$ & $84.4(81.0-87.3)$ & ns \\
\hline \multicolumn{7}{|c|}{ Dementia coding } \\
\hline sensitivity & $56.0(34.9-75.6)$ & $45.2(27.3-64.0)$ & 73.5 (55.6-87.1) & $69.6(47.1-86.8)$ & $66.7(52.1-79.2)$ & 0.13 \\
\hline specificity & $100(98.3-100)$ & $100(98.4-100)$ & $100(98.4-100)$ & $100(97.4-100)$ & $100(98.7-100)$ & ns \\
\hline ppv & 100 & 100 & 100 & 100 & 100 & ns \\
\hline npv & $95.2(92.7-96.9)$ & $92.5(90.5-94.7)$ & $96.7(94.3-98.1)$ & $95.3(91.6-97.4)$ & $94.2(91.6-96.0)$ & ns \\
\hline
\end{tabular}

on dementia; $\mathrm{n}=14$ ) with F05.8 (other delirium) being least common $(n=5$; Table 3). The proportion coded as F05.9 (delirium unspecified) decreased slightly over the study period from $80.0 \%$ (37.6-96.4) in 2010 to $66.7 \%$ (54.1-77.3) in 2018 but differences were nonsignificant. In patients with delirium without comorbid dementia, the vast majority (59/71) received F05.9 (delirium unspecified), and none was given a dementia code (Table 3 ).

In the 100 patients with clinical diagnosis of delirium superimposed on dementia, overall, 74 had at least one delirium or dementia code but only 30 received both a dementia code and a delirium code with 35 being coded as dementia only and nine as delirium only (Table 3). However, there were improvements in coding for delirium on dementia over the study period: 0/11 patients with clinically diagnosed delirium on dementia were coded for both delirium and dementia in 2010 and only 2/18 (11.1\%) in 2012 vs $6 / 13(46.2 \%)$ in 2016 and $19 / 34$ (55.8\%) in 2018. Use of the F05.1 (delirium superimposed on dementia) code also increased from 1/2 in 2012 and 1/6 in 2016 to 16/19 of patients coded for both delirium and dementia in the 2017/18 cohort.

In 103 patients with clinical recording of dementia who had a dementia code, the allocated ICD-10 code was most often F03.X (dementia unspecified; $n=63$ ), followed by F01.9 (vascular dementia; $\mathrm{n}=23$ ) and F05.1 (delirium superimposed on dementia; $n=18$ ) with $\mathrm{G} 30.9$ (Alzheimer's disease) being least common ( $\mathrm{n}=9$;
Fig 3. Sensitivity of administrative ICD-10 coding for clinical diagnosis over each study cycle with $95 \%$ confidence limits. a) Delirium coding. b) Dementia coding. ICD-10 = International Classification of Diseases, 10th revision.
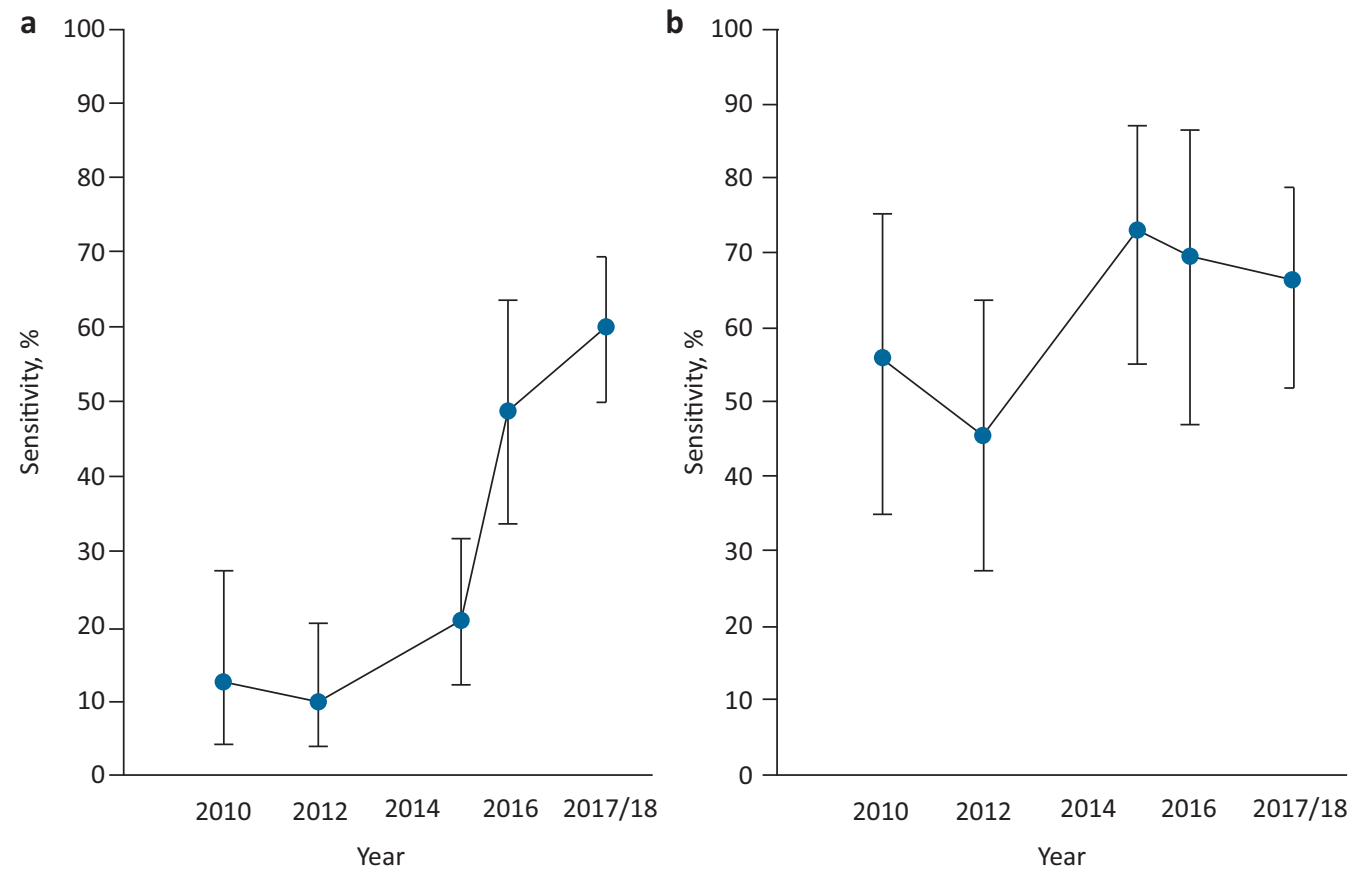


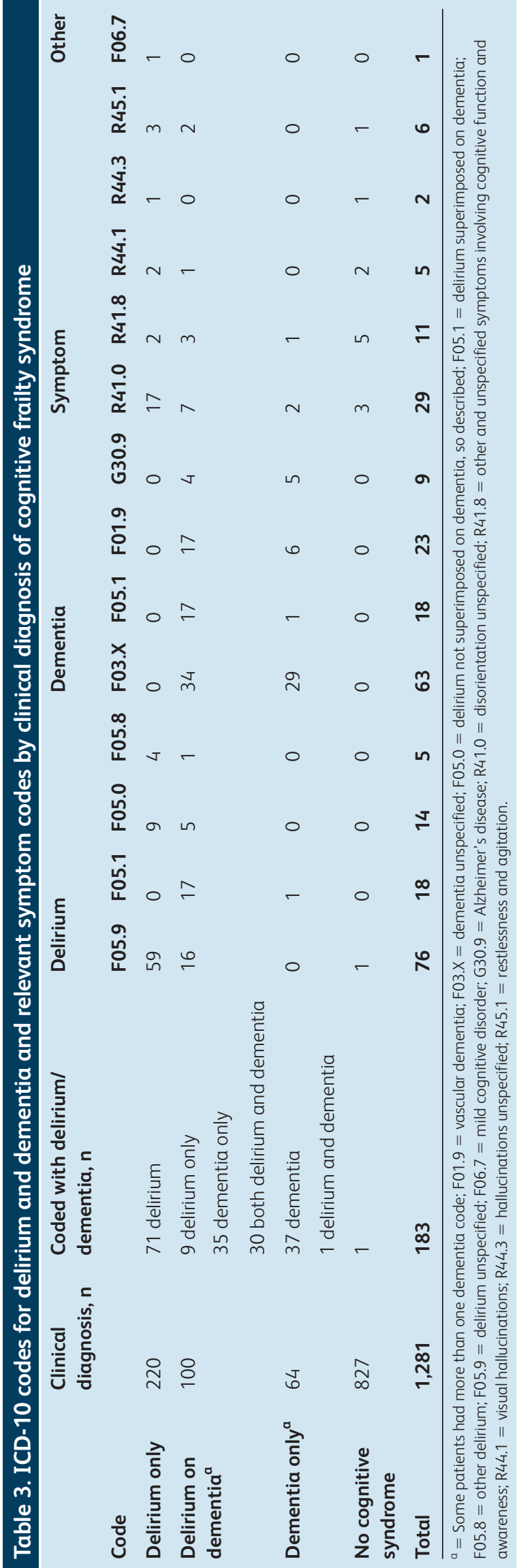

Table 3). Ten patients had two dementia codes (F03.X + G30.9 = 3; G30.9 + F01.9 = 1; F05.1 + F03.X = 6) and in 14/18 with F05.1 (delirium superimposed on dementia), no other dementia code was given. Allocation of F03.X (dementia unspecified) decreased non-significantly over the period of the study from $11 / 14$ (78.6\%) patients coded for dementia in 2010 to $19 / 34$ (55.9\%) of patients coded for dementia in 2018.

Undercoding of cognitive frailty syndromes throughout the study period meant that ICD-10 data under-estimated the total burden of cognitive frailty (Fig 2), although this improved from $19 / 53(35.8 \%$ ) coded with either or both a delirium or dementia code in 2010 to $77 / 120(64.2 \%)$ coded in 2018 . In addition, at the beginning of the study, ICD-10 data implied that most cognitive frailty was dementia alone (14/19 coded cases in 2010) with delirium including delirium on dementia being relatively rare (Fig 2). In the latter part of the study when delirium coding substantially increased, ICD-10 data gave a more representative picture of the relative proportions of the different cognitive frailty sub-types with delirium with or without comorbid dementia being more common (57/77 (74\%) coded cases) than dementia alone (Fig 2).

Fifty patients had an ICD-10 symptom/sign (R) code indicating confusion or hallucinations, three of whom had two such codes (Table 3). The majority of patients with at least one R code (39/50) had a clinically diagnosed cognitive syndrome (Table 3 ) but only $14 / 39$ had an allocated ICD-10 delirium or dementia code. In patients with clinical delirium, 2/24 with R41.0, 2/5 with R41.8, 1/3 with $\mathrm{R} 44.1,0 / 1$ with $\mathrm{R} 44.3$ and $4 / 5$ with $\mathrm{R} 45.1$ also received an ICD10 code for delirium. Therefore 29 patients with clinical delirium received a symptom code without a delirium code. One patient received a code of F06.7 (mild cognitive disorder) who had clinical delirium but no delirium code.

Following completion of our study, we obtained ICD-10 coding data for all adult acute medicine admissions to the OUHFT between July and December 2019 (3,880 patients) in whom 579 (14.9\%; 95\% CI 13.8-16.1) were coded for delirium. This compared with a rate of $60 / 325(18.0 \% ; 14.2-22.7 \%)$ patients coded for delirium in the 2018 cohort managed by our team.

\section{Discussion}

A multicomponent intervention to improve clinical identification and recording of delirium was associated with a six-fold increase in sensitivity of administrative ICD-10 coded data for clinical delirium without sacrificing specificity. As a result, ICD-10 coded data at the end of the intervention period were more representative of the true rate of delirium occurrence and of the relative proportion of delirium vs dementia. Trust-wide rates of coding for delirium in acute medicine 1 year after study completion were similar to those for patients managed by our team at study end suggesting that the impact of the intervention was felt across the hospital system and was sustained. Although calculation of the associated health economic impact was beyond the scope of this study, inclusion of a delirium code may move a given patient from a lower to a higher health resource group potentially doubling the payment received by the hospital for a given admission episode (see supplementary material S2).

To our knowledge, there are no other studies examining delirium coding accuracy from the UK, but the overall low rates of delirium coding we observed at study start are in keeping with previous reports from non-UK settings. ${ }^{3-7}$ However, after the intervention, delirium coding approached that for dementia coding even 
though its fluctuating and transient nature presents diagnostic challenges. Sensitivity of delirium coding rose for both prevalent and incident delirium, although in the later periods, prevalent delirium was coded more often than incident delirium possibly because screening was mandatory on admission. One US study also showed increased delirium coding over time but there was no accompanying gold standard clinical diagnosis for comparison. ${ }^{6}$

Coding sensitivity for clinically recorded dementia diagnosis showed much smaller increases over time possibly because dementia coding was already relatively high. Death certification of dementia has increased slightly over recent years possibly because of greater awareness. ${ }^{25}$ Sensitivity of dementia coding for clinical dementia diagnosis (ie not including undiagnosed (occult) dementia cases) was similar to that reported elsewhere in the UK using a mental healthcare database dementia diagnosis as the gold standard. ${ }^{26}$

The association between a greater total number of ICD-10 codes and coding of both delirium and dementia was unexpected. Coding for dementia on death certificates is less likely in the presence of multiple comorbidities. ${ }^{25}$ However, the positive association we observed suggests that either coders expected delirium and dementia to be more common in those with multimorbidity, or that delirium and dementia codes were allocated more often by administrators who achieved better coding depth overall. It should be noted that senior coding staff remained the same throughout the study and the impact of the intervention might have been reduced with higher staff turnover.

Most delirium was coded as 'delirium unspecified' probably because the clinical documentation did not clearly link the delirium to an underlying medical condition even when one (eg urinary tract infection) was present. We observed changes in coding for delirium occurring in the context of a known dementia diagnosis over the course of the study. In the early years, in particular, under-coding of delirium resulted in patients with delirium on dementia being coded as dementia only, whereas later, most of these patients were assigned F05.1 (delirium superimposed on dementia) probably because of the inclusion of this diagnosis in the Charlson Comorbidity Index and its subsequent use by the coding team. ${ }^{19-21}$ Use of ICD-10 symptom codes for confusion without a code for delirium lessened over the course of the study as delirium coding increased.

'Dementia unspecified' was the most commonly allocated dementia code throughout the study in keeping with data from the USA. ${ }^{27}$ In addition, patients coded as 'delirium superimposed on dementia' often did not have another dementia code. Hospital administrative diagnostic data are therefore likely to be of limited use in subtyping of dementia notwithstanding their use in linkage studies. We did not examine the underlying reasons behind the lack of coding for specific dementia subtypes but information from the coding team indicated that suboptimal clinical documentation is the most likely cause, possibly in part because mental health records are not easily accessible. Coders cannot use information from other admissions or make assumptions about dementia subtype because of accompanying pathology (eg cerebrovascular disease). Where a specific subtype code was used, this was more often for vascular dementia than for Alzheimer's disease in contrast to the known relative rates of the two dementia subtypes in the background population. ${ }^{28}$

Strengths of our study include the large inclusive sample, and determination of delirium coding accuracy on which data are scarce, against careful prospective ascertainment of a clinical gold standard delirium diagnosis in a real-world clinical setting. The multicomponent intervention appeared to have had a substantial and lasting impact on delirium coding across acute medicine consistent with major institutional cultural change. Limitations include the restriction to one institution, but our sample was free of selection bias which may nevertheless affect multicentre studies. We were also not able to examine the impact on delirium coding accuracy in non-medical specialties owing to lack of gold standard delirium diagnosis for comparison. Second, we were not able to examine which specific aspects of the intervention were most effective. We suspect that the introduction of the cognitive screen and meetings with the coding team were important. Third, there was no independent verification of delirium diagnosis but we used established methodology and rates were similar to those reported from comparable populations. ${ }^{1,2,13,14}$ Fourth, we were not able to examine whether under-coding of delirium was caused by suboptimal recording by clinicians or by coding omissions. Accurate clinical documentation is likely to be key, but the associations of delirium/dementia codes and overall coding depth suggests a possible effect of coder experience/engagement. Fifth, we used a classification of cognitive frailty restricted to purely cognitive phenotypes corresponding to ICD-10 coding categories used for hospitalised patients. Other classifications of cognitive frailty exist, including some that combine physical and cognitive deficits, but these are generally designed for use in community settings and do not reflect the dynamic nature of cognition in the context of acute illness. $^{29}$

\section{Conclusion}

Our study demonstrates that substantial (six-fold) improvements in sensitivity of administrative ICD-10 diagnostic coding for delirium can be achieved through a multicomponent intervention involving structured patient assessment, feedback via governance processes and education inclusive of the coding team, without losing diagnostic specificity. Further studies are required to determine the long-term impact beyond 1 year and whether coding for delirium can be further improved in the future. These findings have important implications for the quality of hospital administrative diagnostic coding in general and thereby casemix adjustment, appropriate tariff allocation and remuneration, service planning and the examination of time trends. Finally, ICD-10 coded data for delirium, and therefore HES data, should be used with caution in comparisons of delirium rates across different time periods, services and institutions and in estimating the burden, associates and outcomes of delirium in hospital cohorts.

\section{Summary box}

What is known

Delirium is prevalent in patients with unplanned hospital admission and is associated with high nursing needs, increased costs, death, dependency and future dementia. Despite its importance, available data suggest that hospital administrative coding of delirium is poor. Lack of coding for delirium leads to reduced hospital reimbursement payments (tariff), suboptimal case-mix adjustment and service planning, as well as underestimation of the cognitive frailty burden in hospital cohorts. 
What is the question

We wished to determine the impact of a multicomponent intervention to improve the identification and documentation of cognitive frailty on the sensitivity and specificity of hospital administrative coding for delirium versus the gold standard of prospectively ascertained clinical delirium diagnosis.

What was found

The multicomponent intervention had a substantial impact on the accuracy of administrative coding for delirium: Sensitivity of ICD-10 coded data for the Gold standard clinical diagnosis of delirium increased six-fold from $12.8 \%$ in 2010 to $60.2 \%$ in 2018 while specificity remained at $>99 \%$ throughout. The effect of the intervention was maintained trust-wide at 1 year after the study ended.

What is the implication for practice now?

System-wide interventions can significantly improve delirium coding without increasing false positive coding rates. Any associated costs should be offset by gains in remuneration, better case-mix adjustment and more informed service planning commissioning and health policy. Our findings have implications for hospital coding in general: coding accuracy can be improved by relatively simple measures, even in diagnostically challenging disorders.

\section{Supplementary material}

Additional supplementary material may be found in the online version of this article at www.rcpjournals.org/clinmedicine:

S1 - Cognitive screens delivered via the paper clerking pro forma and via the electronic patient record.

S2 - Sample calculation to illustrate how inclusion of a delirium code may change the tariff received by a hospital for a given patient episode.

\section{Funding}

Dr Pendlebury is supported by the National Institute for Health Research Oxford Biomedical Research Centre. There was no specific funding for this study.

\section{Acknowledgements}

We would like to thank Chris Middlemass, OUHFT senior coding improvement manager and the OUHFT information team for their help with this study.

\section{References}

1 Inouye SK, Westendorp RG, Saczynski JS. Delirium in elderly people. Lancet 2014;383:911-22.

2 Pendlebury ST, Lovett NG, Smith SC et al. Observational, longitudinal study of delirium in consecutive unselected acute medical admissions: age-specific rates and associated factors, mortality and re-admission. BMJ Open 2015;5:e007808.

3 Katznelson R, Djaiani G, Tait G et al. Hospital administrative database underestimates delirium rate after cardiac surgery. Can J Anaesth 2010;57:898-902.

4 Boustani M, Baker MS, Campbell N et al. Impact and recognition of cognitive impairment among hospitalized elders. J Hosp Med 2010;5:69-75.
5 Hope C, Estrada N, Weir C et al. Documentation of delirium in the VA electronic health record. BMC Res Notes 2014;7:208.

6 McCoy TH Jr, Snapper L, Stern TA, Perlis RH. Underreporting of delirium in statewide claims data: implications for clinical care and predictive modeling. Psychosomatics 2016;57:480-8.

7 McCoy TH Jr, Chaukos DC, Snapper LA et al. Enhancing delirium case definitions in electronic health records using clinical free text. Psychosomatics 2017;58:113-20.

8 World Health Organization. The ICD-10 classification of mental and behavioural disorders: clinical descriptions and diagnostic guidelines. Geneva: WHO, 2019.

9 Nouraei SA, Hudovsky A, Frampton AE et al. A study of clinical coding accuracy in surgery: implications for the use of administrative big data for outcomes management. Ann Surg 2015;261:1096-107.

10 Sabia S, Dugravot A, Dartigues JF et al. Physical activity, cognitive decline, and risk of dementia: 28-year follow-up of Whitehall II cohort study. BMJ 2017;357:j2709.

11 Pendlebury ST, Klaus SP, Mather M, de Brito M, Wharton R. Routine cognitive screening in older patients admitted to acute medicine: Abbreviated Mental Test Score (AMTS) and subjective memory complaint versus Montreal Cognitive Assessment and IQCODE. Age Ageing 2015:44:1000-5.

12 Pendlebury ST, Lovett N, Smith SC et al. Delirium risk stratification in consecutive unselected admissions to acute medicine: validation of externally derived risk scores. Age Ageing 2016;45:60-5.

13 Pendlebury ST, Lovett NG, Smith SC, Wharton R, Rothwell PM. Delirium risk stratification in consecutive unselected admissions to acute medicine: validation of a susceptibility score based on factors identified externally in pooled data for use at entry to the acute care pathway. Age Ageing 2017:46:226-31.

14 Phelps A, Kingston B, Wharton RM, Pendlebury ST. Routine screening in the general hospital: what happens after discharge to those identified as at risk of dementia? Clin Med 2017;17:395-400.

15 Inouye SK, van Dyck CH, Alessi CA et al. Clarifying confusion: the confusion assessment method. A new method for detection of delirium. Ann Intern Med 1990;113:941-8.

16 Hodkinson HM. Evaluation of a mental test score for assessment of mental impairment in the elderly. Age Ageing 1972;1:233-8.

17 Folstein MF, Folstein SE, McHugh PR. 'Mini-mental state'. A practical method for grading the cognitive state of patients for the clinician. J Psychiatr Res 1975;12:189-98.

18 American Psychiatric Association. Diagnostic and statistical manual of mental disorders (DSM-IV). Washington: American Psychiatric Association, 1994.

19 Morandi A, Davis D, Bellelli $\mathrm{G}$ et al. The diagnosis of delirium superimposed on dementia: an emerging challenge. J Am Med Dir Assoc 2017;18:12-8.

20 Charlson M, Szatrowski TP, Peterson J, Gold J. Validation of a combined comorbidity index. J Clin Epidemiol 1994;47:1245-51.

21 Dr Foster Intelligence. Understanding HSMRs: A toolkit on hospital standardised mortality ratios. London: Dr Foster, 2014. https://pdfs. semanticscholar.org/4d5e/5abde666f78f27563382d40443d4bc f59e24.pdf [Accessed 18 May 2020].

22 NHS England. Dementia Assessment and Referral. NHS. www. england.nhs.uk/statistics/statistical-work-areas/dementia [Accessed 18 May 2020].

23 Ogrinc G, Davies L, Goodman D et al. SQUIRE 2.0 (Standards for QUality Improvement Reporting Excellence): revised publication guidelines from a detailed consensus process. BMJ Qual Saf 2016;25:986-92.

24 von Elm E, Altman DG, Egger M et al. The Strengthening the Reporting of Observational Studies in Epidemiology (STROBE) statement: guidelines for reporting observational studies. J Clin Epidemiol 2008;61:344-9.

25 Gao L, Calloway R, Zhao E, Brayne C, Matthews FE. Accuracy of death certification of dementia in population-based samples of older people: analysis over time. Age Ageing 2018;47:589-94. 
26 Sommerlad A, Perera G, Singh-Manoux A et al. Accuracy of general hospital dementia diagnoses in England: Sensitivity, specificity, and predictors of diagnostic accuracy. Alzheimers Dement 2018;14:933-43.

27 Butler D, Kowall NW, Lawler E, Michael Gaziano J, Driver JA. Underuse of diagnostic codes for specific dementias in the Veterans Affairs New England healthcare system. J Am Geriatr Soc 2012;60:910-5.

28 Lobo A, Launer LJ, Fratiglioni L et al. Prevalence of dementia and major subtypes in Europe: A collaborative study of populationbased cohorts. Neurologic Diseases in the Elderly Research Group. Neurology 2000;54:S4-9.
29 Kelaiditi E, Cesari M, Canevelli M et al. Cognitive frailty: rational and definition from an (I.A.N.A.II.A.G.G.) international consensus group. J Nutr Health Aging 2013;17:726-34.

Address for correspondence: Dr Sarah Pendlebury, Centre for Prevention of Stroke and Dementia, Wolfson Building, John Radcliffe Hospital, Oxford OX3 9DU, UK. Email: sarah.pendlebury@ndcn.ox.ac.uk

\section{Royal College of Physicians}

\section{Rebuilding the NHS RCP priorities for the resetting of services}

The RCP has set out nine key priorities for the resetting and rebuilding of NHS services, now that the initial COVID-19 peak has passed.

The publication covers the role of the medical specialties and emphasises that our healthcare system must not simply return to how it was pre-pandemic. From routine care to reducing health inequalities, particularly for people from ethnic minority backgrounds, we have the opportunity to embed long-term improvements.

Download the document: www.rcplondon.ac.uk/rebuilding-the-NHS

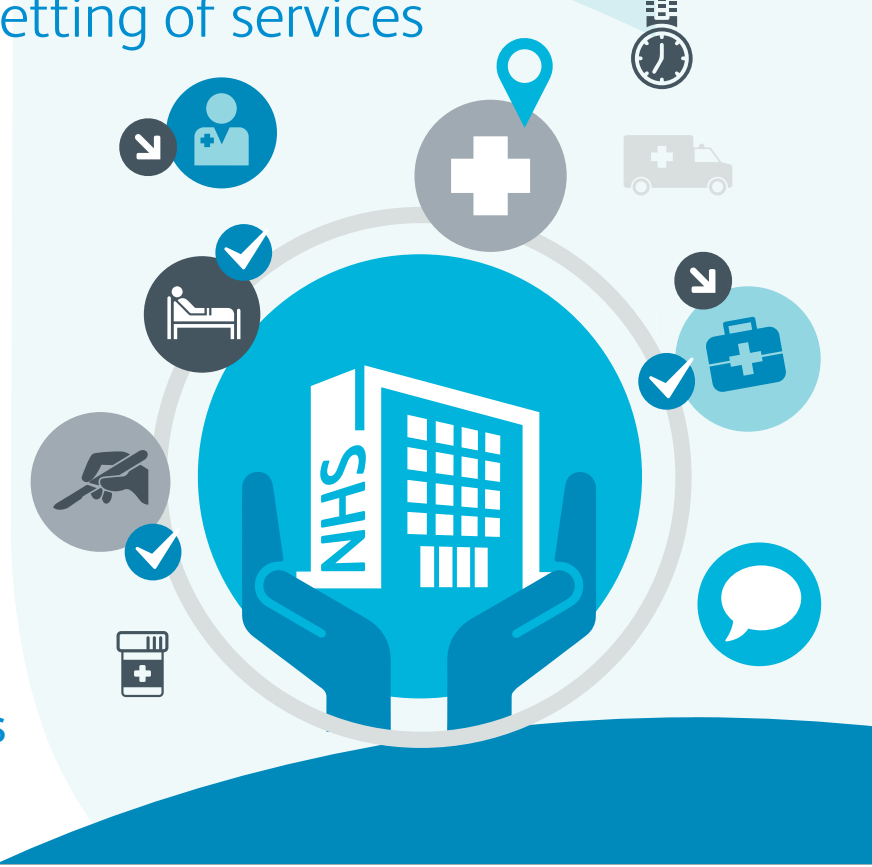

